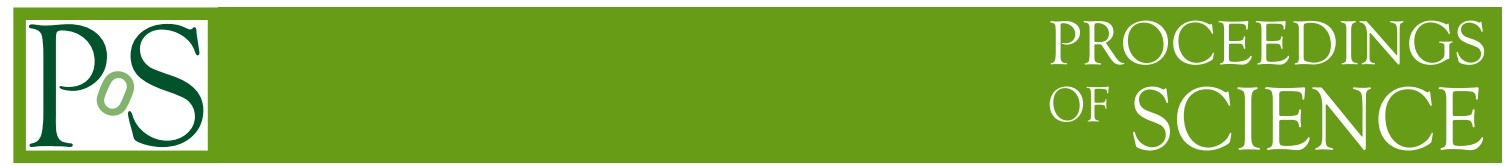

\title{
Multi-wavelength signals from Dark Matter annihilation at the Galactic Center
}

\author{
Regis Marco* \\ International School for Advanced Studies (SISSA/ISAS) and INFN, Trieste. \\ E-mail: regisesissa.it
}

\begin{abstract}
In this proceeding paper we analyze the dark matter annihilation as a source of a multi-wavelength photon spectrum. In particular the source under investigation is located at the Galactic Center. Direct annihilation into photons, gamma-rays from $\pi^{0}$ decay and emissions from radiative processes, as X-rays from Inverse Compton scattering and synchrotron radiation in the $\mathrm{X}$, InfraRed and Radio bands, are discussed. The goal is to point out how the consistency among the level and eventually the spatial profile of the flux at different frequencies is a powerful way to constrain dark matter models.
\end{abstract}

Cargãlse Summer School: Cosmology and Particle Physics Beyond the Standard Models June 30-August 112007

Cargese, France

\footnotetext{
* Speaker.
} 
The adjective dark in the name Dark Matter (DM) pictorially connotes the very tiny interaction between DM and light. Dark and not black since the DM could be not completely decoupled from photons; rather, in some models, this coupling is unavoidable at quantum level. Moreover it's not completely excluded that a tiny fraction of the whole dark matter content of the Universe is charged or millicharged or that the DM has an electric or magnetic dipole moment. Nevertheless all these possibilities are very strongly constrained by experimental data [1]. The adjective dark turns out to be very appropriate. From this simple observation, it does not look very promising to investigate the nature of DM analyzing the photon spectrum originated from its annihilation.

The class of dark matter candidates under investigation in this paper is the WIMP class. A WIMP is a particle that was in thermal equilibrium in the primordial Universe, meaning that it must have some (weak) interactions with particles of the bath. Thus, in this case, it's natural to expect couplings to photons at quantum level and secondary photons emitted by the products of the dark matter annihilation. The photon spectrum that we are looking for is essentially related to these two phenomena.

To enhance the probability of copious dark matter annihilations, we have to look at regions where the dark matter density is very large. Moreover, in order to identify such flux, we have to face the astrophysical background composed by any other photon sources in the region of investigation. Thus, to disentagle the dark matter signal, we have to concentrate on objects where such background is faint, and/or on the particular features of the spectrum generated by DM.

There are few astrophysical objects where we restrict this hunt. From the point of view of brightness, the Galactic Center is probably the most promising source, in particular if the dark matter profile of the Milky Way is cuspy. The drawback is that a source, which is named Sgr A* and is intimely related to the Milky Way supermassive black hole (SMBH), shows robust spectra in the radio, infrared, $\mathrm{X}$ and gamma bands, hiding an eventual dark matter source.

One way to overcome this problem is to look for a monoenergetic spectral signature [2]. Indeed a WIMP could annihilate into photon pairs (or $\gamma-Z$ ) at one loop level, thus generating a line in the gamma spectrum at energy $E=M_{D M}$ (or $E=M_{D M}\left(1-M_{Z}^{2} / 4 M_{D M}^{2}\right)$ ) which cannot be mimicked by other astrophysical emission processes. In order to detect a line signal under a continuum background, the number of events associated to the first has to overcome the uncertaintes in the events of the latter, in the same bin of energy. It becomes more and more plausible as the resolution of the experiment increases (namely the bin width decreases) or as the statistical and systematic errors decrease. Reducing the statystical errors requires the expansion of the effective area or of the time of exposure. The most powerful dark matter hunters from gamma-rays in the next future will be the space satellite GLAST [3] and the Air Cherenkov Telescope of the CTA project [4]. The first is scheduled to be launched in 2008 and the energy range of detection is approximately $100 \mathrm{MeV}-300 \mathrm{GeV}$, thus covering the "soft"-gamma part of the WIMP spectrum. Its large field of view allows a huge exposure time leading to low expected errors and a very good sensitivity. The CTA project is at an advanced status. The energy range of detection proposed is $10 \mathrm{GeV}-100 \mathrm{TeV}$ (the "hard"-gamma part of the WIMP spectrum) and, basically thanks to a huge effective area, of the order of $1 \mathrm{~km}^{2}$ or even larger in its extreme configuration, it should be able to highly reduce (compared to the Air Cherenkov Telescope of the HESS collaboration [5]) the statistical errors. In both case the energy resolution is approximately $10 \%$.

On the top of a line signal, a gamma-rays continuum component in the spectrum is expected. 
This is generated by decays of $\pi^{0}$ into $\gamma \gamma$. Indeed WIMP annihilations produce primary or secondary quarks, that subsequently hadronize into $\pi^{0}$. With an energy resolution $\gtrsim$ few $\%$ such flux is typically much higher than the line signal. On the other hand it is not easy to unambigously identify the source and, moreover, at the Galactic Center it is hidden by an other continuum astrophysical signal, at least in the range $100 \mathrm{GeV}-50 \mathrm{TeV}$ [5]. The possibility of extending the range of investigation in the soft part by GLAST and of reducing the error bars by CTA could eventually unveil a DM component in this flux.

At lower energy (i.e. starting from hard X-rays) the photon emission by dark matter annihilation is mainly related to radiative processes. Electrons and positrons, primary or secondary generated, could radiate or scatter with photons, producing a predictable continuum flux.

The way of producing electrons is a consequence of the particle physics model in which the WIMP is embedded, while the way of propagating of such electrons and their resulting distribution is regulated by the transport equation (see for example [6]):

$$
\frac{\partial \psi(\vec{r}, p, t)}{\partial t}=q(\vec{r}, p, t)+\vec{\nabla} \cdot\left(D_{x x} \vec{\nabla} \psi-\vec{v} \psi\right) \frac{\partial}{\partial p} p^{2} D_{p p} \frac{\partial}{\partial p} \frac{1}{p^{2}} \psi-\frac{\partial}{\partial p}\left[\dot{p} \psi-\frac{p}{3}(\vec{\nabla} \cdot \vec{v}) \psi\right]
$$

where $\psi$ is the electron/positron distribution, $q$ is the dark matter source, $\dot{p}$ is the momentum loss rate related to radiative processes, $D_{i i}$ are the diffusion coefficients and $\vec{v}$ is the advection/convection velocity. The usual assumption is time independence in the source and equilibrium reached, namely the term on the r.h.s. being 0 .

Inverse Compton scattering on CMB or starlight is the most relevant contribution in the hard $\mathrm{X}$-rays. In this case the signal is produced by ultra-relativistic electrons and the only relevant terms in Eq. 1 are the dark matter source and the energy loss rate; the solution is thus straightforward. Here the main uncertainty (not related to the DM properties) is represented by the starlight spectrum. At lower energy the synchrotron radiation becomes more important than the IC emission. The exact energy at which this happens is mainly set by the strength of the magnetic field in which the electrons propagate. In the case of the Galactic Center, the equipartition between kinetic and magnetic energy in the SMBH accretion flow is a well motivated assumption [7], leading to a magnetic field $B \propto r^{-5 / 4}$ and thus very high in the central part. The synchrotron emission from ultra-relativistic electrons is thus the dominant contribution from and below the (soft) X-rays [8]. When pointing in the direction of the GC, the absorption due to photoeletric effect on dust is very efficient in the soft-X and ultraviolet bands, suppressing the flux. Up to these frequencies, the dark matter source is very narrow, essentially compatible to a point source in any of the present experiment, if a cuspy DM profile is assumed. Thus its flux has to be compatible with the smallest measured source at the GC, namely Sgr A*.

Different is the picture in the Radio and InfraRed bands, where the diffusion and advection/convection effects could be non-negligible [9]. In this case the source is an extended source with a dimension driven by the halo profile, the magnetic field, the diffusion coefficient and the velocity $v$. This is probably the most interesting signal because, for a given dark matter candidate, one can predict not only the radio (or IR) spectrum, but even the spatial profile of the signal. On the other hand this is the most uncertain flux, since we have to know the shape and the strenght of the magnetic field, the expression for the diffusion coefficient and how the eventual advective/convective process unfolds. A detailed study, with the goal of carrying out a less 
model dependent dark matter signal in the radio band, could be promising to constraint or unveil the dark matter properties. In the case of the GC, due to the strong magnetic field, the diffusion term is always negligible and the effects from the adiabatic compression in the SMBH accretion flow are subdominant. Thus the solution of Eq. 1 is again well approximated by taken into account only synchrotron losses. The source extension depends on the halo profile and on the frequency of emission, and it is constrained by measurements of the Sgr A West source or of the whole Sgr A.

An example of the multi-wavelength study sketched in this paper is performed in [10], where signals and possible detection of a WIMP candidate, embedded in an extra dimension model [11] and distributed on a spiky profile, are presented.

In this brief and partial discernment on the multi-wavelength spectrum from dark matter annihilation at the Galactic Center, the goal was to point out the opportunity to consistently test a dark matter model in the whole electromagnetic spectrum, emphasizing the capability of putting contraints also with low energy measurements. An interesting highlight is the possibility of investigating the shape of the DM halo profile through the radio angular signal. However to make more defined constraints, a more detailed and predictive study of the electron/positron propagation at the Galactic Center is needed.

\section{Acknowledgments}

We would like to thank Piero Ullio for useful comments and discussions.

\section{References}

[1] A. Gould, B. T. Draine, R. W. Romani and S. Nussinov, Neutron stars: graveyard of charged Dark Matter, Phys. Lett. B 238 (1990) 337. A. Melchiorri, A. Polosa and A. Strumia, New bounds on millicharged particles from cosmology, Phys. Lett. B 650 (2007) 416 [arXiv:hep-ph/0703144]. K. Sigurdson, M. Doran, A. Kurylov, R. R. Caldwell and M. Kamionkowski, Dark-matter electric and magnetic dipole moments, Phys. Rev. D 70 (2004) 083501 [Erratum-ibid. D 73 (2006) 089903] [arXiv:astro-ph/0406355].

[2] L. Bergstrom, P. Ullio and J. H. Buckley, Observability of gamma rays from dark matter neutralino annihilations in the Milky Way halo, Astropart. Phys. 9 (1998) 137 [arXiv:astro-ph/9712318].

[3] http://www-glast.slac.stanford.edu/software/IS/glast_lat_performance.html

[4] http://www.mpi-hd.mpg.de/hfm/CTA/CTA_home.html

[5] F. Aharonian et al. [The HESS Collaboration], Very high energy gamma rays from the direction of Sagittarius $A^{*}$, Astron. Astrophys. 425 (2004) L13, [arXiv:astro-ph/0408145].

[6] A. W. Strong, I. V. Moskalenko and V. S. Ptuskin, Cosmic-ray propagation and interactions in the Galaxy, Ann. Rev. Nucl. Part. Sci. 57 (2007) 285 [arXiv:astro-ph/0701517].

[7] F. Melia, An accreting black hole model for Sagittarius A*, Ap. J. 387, L25-L28, 1992

[8] L. Bergstrom, M. Fairbairn and L. Pieri, X-ray radiation from the annihilation of dark matter at the galactic center, Phys. Rev. D 74 (2006) 123515 [arXiv:astro-ph/0607327].

[9] R. Aloisio, P. Blasi and A. V. Olinto, Neutralino annihilation at the galactic center revisited, JCAP 0405 (2004) 007 [arXiv:astro-ph/0402588].

[10] M. Regis and P. Ullio, in preparation.

[11] M. Regis, M. Serone and P. Ullio, A dark matter candidate from an extra (non-universal) dimension, JHEP 0703 (2007) 084, [arXiv:hep-ph/0612286]. 